\title{
Role of the Raman gain in the noise dynamics of all-normal dispersion silica fiber supercontinuum generation
}

\author{
Gonzalo, Iván Bravo; Bang, Ole
}

Published in:

Journal of the Optical Society of America B: Optical Physics

Link to article, DOI:

10.1364/JOSAB.35.002102

Publication date:

2018

Document Version

Peer reviewed version

Link back to DTU Orbit

Citation (APA):

Gonzalo, I. B., \& Bang, O. (2018). Role of the Raman gain in the noise dynamics of all-normal dispersion silica fiber supercontinuum generation. Journal of the Optical Society of America B: Optical Physics, 35(9), 2102-2110. https://doi.org/10.1364/JOSAB.35.002102

\section{General rights}

Copyright and moral rights for the publications made accessible in the public portal are retained by the authors and/or other copyright owners and it is a condition of accessing publications that users recognise and abide by the legal requirements associated with these rights.

- Users may download and print one copy of any publication from the public portal for the purpose of private study or research.

- You may not further distribute the material or use it for any profit-making activity or commercial gain

- You may freely distribute the URL identifying the publication in the public portal 


\title{
Role of the Raman gain in the noise dynamics of all- normal dispersion silica fiber supercontinuum generation
}

\author{
IVÁn BRAVo GonzALO ${ }^{1, *}$ ANd Ole BANG ${ }^{1,2}$ \\ ${ }^{1}$ Technical University of Denmark, Department of Photonics Engineering, Kgs. Lyngby, 2800, Denmark \\ ${ }^{2}$ NKT Photonics A/S, Birkerød, 3460, Denmark \\ *Corresponding author: ibag@fotonik.dtu.dk
}

Received XX Month XXXX; revised XX Month, XXXX; accepted XX Month XXXX; posted XX Month XXXX (Doc. ID XXXXX); published XX Month XXXX

\begin{abstract}
We theoretically and numerically study the influence of the Raman gain profile on the noise dynamics of the supercontinuum generation in a standard all-normal dispersion silica fiber using the scalar generalized nonlinear Schrödinger equation. In particular, we investigate the effect of the different secondary resonance gain peaks on the evolution of the SC coherence by comparing the coherence obtained when using the measured Raman gain of silica with that obtained using different analytical approximations. We demonstrate that the strongest secondary peak at $14.8 \mathrm{THz}$ has a significant influence in that it leads to an early development of a decoherence band on the long wavelength side of the SC. In contrast, the decoherence is strongly dominated by the short wavelength side below the pump for all analytical models not taking this $14.8 \mathrm{THz}$ gain peak into account. We demonstrate that this is due to the $14.8 \mathrm{THz}$ peak being spectrally much narrower than the other gain peaks. (C) 2018 Optical Society of America
\end{abstract}

OCIS codes: (320.6629) Supercontinuum generation; (190.4370) Nonlinear optics, fibers; (190.5650) Raman effect.

\section{INTRODUCTION}

Supercontinuum (SC) sources using all-normal dispersion (ANDi) silica fibers are promising candidates to combine the broadband performance of the anomalous dispersion pumped SC with excellent coherence and low noise [1-3]. This is because noise sensitive mechanisms, such as scalar modulation instability (MI) and soliton collisions can be suppressed [4-6]. Coherent ANDi SC based on selfphase modulation (SPM) and optical wave breaking (OWB), which are coherent processes [6-7], can be achieved under certain conditions. For instance, the pump pulse duration and fiber length are important parameters in the coherence of the SC, and the limits were explained by Heidt et al. [8] assuming scalar single-polarization propagation. In the regime of low absolute dispersion and high pump peak powers, the noise-seeded dynamics from stimulated Raman scattering (SRS) can be suppressed by parametric four-wave mixing (FWM) resulting in a coherent SC when the fiber length is shorter than the coherence length [8]. When longer fiber lengths are used, exceeding the coherence length, decoherence takes place due to noise arising from SRS. In these conditions, ANDi SC generated with short femtosecond pump pulses ( $\$ 600 \mathrm{fs}$ ) is the broadest possible and could be coherent up to around $1 \mathrm{~m}$ for $100 \mathrm{~kW}$ peak pump power [8]. However, for picosecond pump pulses $(\gtrsim 1.6 \mathrm{ps})$ the degradation due to SRS occurs before the broadest possible SC is generated and shorter fibers are needed for coherent SC. Furthermore, when polarization effects are taken into account, polarization modulation instability (PMI) strongly reduces the regime of operation for coherent ANDi SC [9]. For instance, the broadest possible and coherent SC at $1 \mathrm{~m}$ of a standard ANDi fiber could only be achieved with pump pulses shorter than 120 fs for 44 $\mathrm{kW}$ [9].

In this paper, the polarization effects are neglected in order to better focus on the interplay between FWM and SRS as the main mechanism that affects the coherence properties in ANDi SC. We theoretically and numerically investigate the role that the Raman gain profile plays in the strength and spectral distribution of the noise and the overall dynamics of the ANDi SC. The simulations are done using the scalar GNLSE model, and the noise performance is investigated with the first-order coherence function using $600 \mathrm{fs}$ and $1.6 \mathrm{ps}$ long pump pulses as appropriate examples.

In particular, we investigate the effect of the different secondary resonance Raman gain peaks on the evolution of the SC coherence by comparing the coherence obtained when using the measured Raman gain with that obtained using different analytical approximations. We demonstrate that the strongest and spectrally narrow secondary peak at $14.8 \mathrm{THz}$ has a significant influence in that it leads to an early development of a decoherence band on the long wavelength side of the $\mathrm{SC}$. In contrast, the decoherence is strongly dominated by the short wavelength side below the pump for all analytical models not taking this $14.8 \mathrm{THz}$ gain peak into account. 


\section{NUMERICAL MODEL}

\section{A. Generalized nonlinear Schrödinger equation and coherence calculation}

We use the scalar approximation of the generalized nonlinear Schrödinger equation (GNLSE), where single-mode single-polarization is assumed [4]. In this way, polarization effects are neglected and SRS is known to be the main source of noise [8]. The GNLSE is solved in the frequency domain using the interaction picture formulation and a fourth-order Runge-Kutta method [10]. The GNLSE is written as

$$
\begin{aligned}
\frac{\partial \tilde{A}(\Omega, z)}{\partial z} & =i\left[\beta(\omega)-\left[\beta\left(\omega_{0}\right)+\beta_{1}\left(\omega_{0}\right) \Omega\right]\right] \tilde{A}(\Omega, z)-\frac{\alpha(\omega)}{2} \tilde{A}(\Omega, z) \\
& +i \gamma\left(1+\frac{\Omega}{\omega_{0}}\right) \cdot \mathrm{F}\left\{\left(1-f_{R}\right) A(t, z)|A(t, z)|^{2}\right. \\
& \left.+f_{R} A(t, z) \cdot \mathrm{F}^{-1}\left\{\tilde{h}_{R}(\Omega) \cdot \mathrm{F}\left\{|A(t, z)|^{2}\right\}\right\}\right\},
\end{aligned}
$$

where $A(\mathrm{t}, z)$ is the field envelope in the time domain and $\tilde{A}\left(\Omega=\omega-\omega_{0}, z\right)$ is its Fourier transform. The fiber dispersion, included in $\beta(\omega)-\beta\left(\omega_{0}\right)+\beta_{1}\left(\omega_{0}\right) \Omega$, and total loss $\alpha(\omega)$ are taken into account in the first line of Eq. (1) and shown in Fig. 1(a). The dispersion profile of the ANDi silica fiber (corresponding to the widely used and commercially available NL-1050-NEG-1 from NKT Photonics) and the confinement loss of the fiber are calculated with COMSOL Multiphysics. The total loss includes the confinement loss and the material loss of silica [11]. The nonlinear part includes a constant nonlinear parameter $\gamma=34(\mathrm{~W} \mathrm{~km})^{-1}$ for simplicity. The Raman response function of silica in the frequency domain, $\tilde{h}_{R}(\Omega)$, is also added. The measured Raman gain profile and two of the analytical Raman gain models used in this study are shown in Fig. 1(b), which is explained more in detail in the next section.
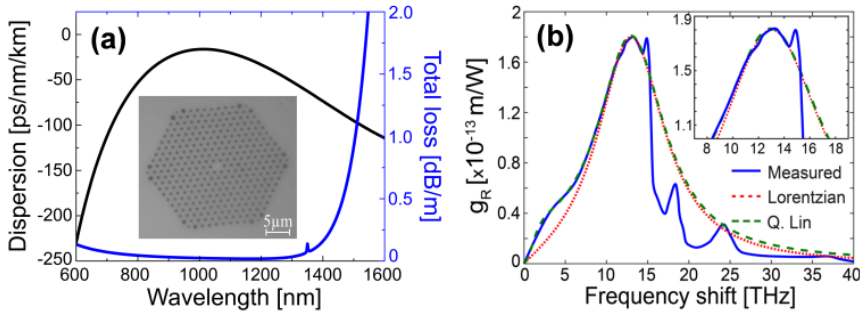

Fig. 1. (a) Calculated dispersion (black) and total loss (blue), including material and confinement loss, of the ANDi fiber $(\Lambda=1.44 \mu \mathrm{m}$, $d / \Lambda=0.39$ ). (b) Raman gain measured at $\lambda_{p}^{R}=526 \mathrm{~nm}[12,13]$ (full blue), Lorentzian model (dotted red) and Q. Lin model (dashed green) used in the simulations. The inset in (b) shows the peak at around 14.8 $\mathrm{THz}$ in the measured Raman gain, which is missing in the analytical models.

The initial conditions for all the simulations are the following: the pump is a Gaussian pulse with central wavelength at $1 \mu \mathrm{m}$ and field envelope in the time domain $A(0, T)=\sqrt{P_{0}} \exp \left[-T^{2} / 2 T_{0}^{2}\right]$. The peak power $P_{0}$ is fixed to $100 \mathrm{~kW}$ and $T_{0}=T_{F W H M} /(2 \sqrt{\ln 2})$, where $T_{F W H M}$ is the full width at half maximum of the power.
To evaluate the coherence properties of the SC we include quantum noise semi-classically in the input field envelope using the one-photon per mode model $[4,14]$. To characterize the shot-to-shot fluctuations, an ensemble of 20 simulations with different noise seeds is used to calculate the first-order coherence at zero-path difference given by $[4,15,16]$

$$
\left|g_{12}{ }^{(1)}(\lambda)\right|=\mid \frac{\left\langle\tilde{A}_{i}^{*}(\lambda) \tilde{A}_{j}(\lambda)\right\rangle_{i \neq j}}{\sqrt{\left.\left.|| \tilde{A}_{i}(\lambda)\right|^{2}\right\rangle\left\langle\left|\tilde{A}_{j}(\lambda)\right|^{2}\right\rangle}},
$$

where angle brackets indicate an ensemble average over independent SC pairs $\left[\tilde{A}_{i}(\lambda), \tilde{A}_{j}(\lambda)\right]$ generated from the 20 simulations with different quantum noise.

\section{B. Raman gain of silica}

The nonlinear response function of silica can be written as

$$
\begin{aligned}
R(\tau) & =\left(1-f_{R}\right) \delta(\tau)+f_{R} h_{R}(\tau) \\
& =\left(1-f_{R}\right) \delta(\tau)+f_{R}\left[R_{a}(\tau)+R_{b}(\tau)\right],
\end{aligned}
$$

where $f_{R}$ represents the fractional Raman contribution [17]. The first term is the instantaneous electronic response and the second is the delayed nuclear response of the material. In Eq. (3), the delayed Raman response $h_{R}(\tau)$ consists of the isotropic $R_{a}(\tau)$ and anisotropic $R_{b}(\tau)$ contributions $[5,12,18]$. The copolarized Raman gain is given by $g_{R}(\Omega)=2 \gamma^{\prime} f_{R} \operatorname{Im}\left[\tilde{h}_{R}(\Omega)\right][5,12]$, where $\gamma^{\prime}=\gamma A_{\text {eff }}=2 \pi n_{2} / \lambda_{p}^{R}$ with $n_{2}=2.6 \times 10^{-20} \mathrm{~m}^{2} / \mathrm{W}$. The orthogonally polarized part of the Raman gain is excluded in the simulations. Figure 1(b) shows 3 of the Raman response functions used in the study, which are explained in the following:

- Lorentzian model: this is the simplest single-resonance model, which only considers the isotropic part $\left(R_{b}(\tau)=0\right)$. This model has been used extensively in the literature on nonlinear pulse propagation in optical fibers and has the form [19]

$$
h_{R}(\tau)=\tau_{1}\left(\tau_{1}^{-2}+\tau_{2}^{-2}\right) \exp \left(-\tau / \tau_{2}\right) \sin \left(\tau / \tau_{1}\right),
$$

where $\tau_{1}=12.2 \mathrm{fs}, \tau_{2}=32 \mathrm{fs}$ and $f_{R}=0.2$ [19]. This model overestimates the Raman gain in the frequency shift range above 15 THz and underestimates it below $10 \mathrm{THz}$ as shown in Fig. 1(b).

- Q. Lin model: this model corrects the underestimation below 10 $\mathrm{THz}$ of the Lorentzian model by including the anisotropic contribution to the copolarized Raman gain, and is given by [12]

$$
\begin{aligned}
& h_{R}(\tau)=f_{a} h_{a}(\tau)+\left[f_{c} h_{a}(\tau)+f_{b} h_{b}(\tau)\right], \\
& h_{a}(\tau)=\tau_{1}\left(\tau_{1}^{-2}+\tau_{2}{ }^{-2}\right) \exp \left(-\tau / \tau_{2}\right) \sin \left(\tau / \tau_{1}\right), \\
& h_{b}(\tau)=\left[\left(2 \tau_{b}-\tau\right) / \tau_{b}{ }^{2}\right] \exp \left(-\tau / \tau_{b}\right),
\end{aligned}
$$

where $\tau_{b}=96 \mathrm{fs}, f_{a}=0.75, f_{b}=0.21, f_{c}=0.04$, and $f_{R}=0.245$. The isotropic part $R_{a}(\tau)=f_{a} h_{a}(\tau)$ corresponds to the Lorentzian model, and the anisotropic part of the Raman gain is included as $R_{b}(\tau)=f_{c} h_{a}(\tau)+f_{b} h_{b}(\tau)$, which dominates in the low frequency region. In this way, the Boson peak at around $3 \mathrm{THz}$ is considered, in contrast to the Lorentzian model (Fig. 1(b)) [12]. 
- Measured Raman gain: The Raman gain of silica measured at $\lambda_{p}^{R}=526 \mathrm{~nm}$ is shown in Fig. 1(b) [12,13], which differs from the Q. Lin model at frequency shifts above $14 \mathrm{THz}$. In particular, the measured Raman gain has a strong sharp peak at around $14.8 \mathrm{THz}$, which is not included in the Lorentzian and Q. Lin models. Similar Raman gain profiles have been measured at other pump wavelengths $[5,20]$. The fractional contribution used here is $f_{R}=0.21$. This value is chosen instead of the commonly used 0.18 [17] because it allows us to have a fair comparison between Raman gains by normalizing them to the Raman gain peak at $13.2 \mathrm{THz}$. Other values of the fractional contribution can be also found in the literature [21].

In the Raman response functions summarized above, the $f_{R}$ is estimated for each model by fitting the Raman gain $g_{R}(\Omega)$ to the measured peak Raman gain $g_{R}\left(\Omega_{R}=13.2 \mathrm{THz}\right) \approx 1.81 \cdot 10^{-13} \mathrm{~m} / \mathrm{W}$. This normalization to the measured peak Raman gain (Fig. 1(b)), which is needed in order to implement a fair comparison between the different Raman gains, was also used before in the literature $[5,12]$. In addition, the Raman response function is always normalized in the simulations, such that $\int_{0}^{\infty} h_{R}(t) d t=1[19]$.

\section{NOISE DYNAMICS}

\section{A. Mixed parametric-Raman gain}

As shown by Heidt at al [8], the Raman gain in ANDi SC can be suppressed under certain conditions due to the coupling between FWM and Raman. The suppression of the Raman gain by FWM is well known and it has been investigated before theoretically and experimentally [22-24]. The effective Raman gain when the FWM and SRS are taken into account is called mixed parametric-Raman (MPR) gain, and is given by [8, 22-24]

$$
g^{*}(\Omega)=2 \gamma P_{0} \operatorname{Re}\{\sqrt{K(2 q-K)}\}
$$

where $K=-\Delta \beta /\left(2 \gamma \mathrm{P}_{0}\right)$ is the linear phase mismatch normalized to the nonlinear contribution to the mismatch, with $\Delta \beta=\beta_{2} \Omega^{2}+\beta_{4} \Omega^{4} / 12+\ldots$ and $q=\left(1-f_{R}\right)+f_{R} \tilde{h}_{R}(-\Omega)$. The dispersion values for the ANDi fiber shown in Fig. 1(a) are $\beta_{2}=8.67 \cdot 10^{-27} \mathrm{~s}^{2} / \mathrm{m}$ and $\beta_{4}=1.93 \cdot 10^{-55} \mathrm{~s}^{4} / \mathrm{m}$ at $1 \mu \mathrm{m}$, which makes $\Delta \beta$ positive and thus $K<0$. Let us summarize the limits of the MPR gain for normal dispersion pumping at $1 \mu \mathrm{m}$ and $\Delta \beta$ positive: When $K \rightarrow 0$, corresponding to high pump peak power or pumping close to the zero dispersion, the MPR gain is reduced, and completely canceled for $K=0$. In addition, when SRS is negligible ( $f_{R}=0 \rightarrow q=1$ ), the MPR gain is zero because $K<0$. However, pure parametric gain can be obtained when $\beta_{4}<0$ [23] for frequency shifts at which $K$ becomes positive. Therefore, it is important to consider $\beta_{4}$ in the calculations of the MPR gain in normal dispersion as it can lead to MI sidebands for some frequency shifts, depending on the dispersion values. On the other hand, when $K \rightarrow \pm \infty$ there is complete phase mismatch and thus FWM has no influence. Consequently, the MPR gain becomes $g_{R}^{*}=g^{*}(\mathrm{~K} \rightarrow \pm \infty)=-2 \gamma P_{0} \operatorname{Im}\{q\}=2 \gamma P_{0} \mathrm{f}_{R} \operatorname{Im}\left\{\tilde{h}_{R}(\Omega)\right\}$, which we denote $g_{R}^{*}$ because it is directly proportional to the pure measured Raman gain $g_{R}^{*}=\left(P_{0} / A_{\mathrm{eff}}\right) g_{R}$.
The MPR gain suppression can be estimated for the parameters used here by calculating the linear phase mismatch parameter at the Raman peak $\Omega=2 \pi \cdot 13.2 \mathrm{THz}$, which gives $K \approx-0.0089$. From this value, we expect FWM-induced suppression of the Raman gain in that the MPR gain length $L_{R}^{*}=1 / g^{*}=6.89 \mathrm{~mm}$ is more than 10 times longer than the corresponding gain length that would be obtained when FWM is neglected, $L_{R}^{*}=1 / g_{R}{ }^{*}=0.51 \mathrm{~mm}$.

Figure 2 shows the MPR gain normalized with $2 \gamma P_{0}$ for the measured Raman gain. First, we observe that the MPR gain $g^{*}$ is drastically reduced in comparison to the corresponding pure Raman gain $g_{R}^{*}$, as expected from the calculation of the MPR gain at $13.2 \mathrm{THz}$. We can also see that in contrast to $g_{R}^{*}$, in which the maximum gain is at $13.2 \mathrm{THz}$, the MPR gain $g^{*}$ has a maximum around $14.8 \mathrm{THz}$. This can be explained by the fact that the reduction of the Raman gain by FWM is frequency dependent through the parameters $K$ and $q$. For a given Raman gain, the value of $|K|$ increases with frequency shift and therefore gain suppression is lower at higher frequency shifts, as shown in the inset of Fig. 3.

Figure 3 shows the MPR gain normalized with $2 \gamma P_{0}$ for the measured Raman gain and the Lorentzian and Q. Lin models. The parameter values are the same as in Fig. 2. We observe that the Boson peak at around $3 \mathrm{THz}$, in the measured Raman gain and the Q. Lin model (Fig. 1(b)), is completely suppressed by FWM due to the low value of $|K|$ here (see inset). As a result, the shape of the MPR gain is the same for the Q. Lin and Lorentzian models, but slightly higher for all frequency shifts with the Q. Lin model due to the chosen normalization of the Raman response. Thus, we would expect to observe higher noise when using the Q. Lin model compared to the Lorentzian model. The MPR gain for the measured Raman gain is similar to both analytical models at frequency shifts lower than around $14 \mathrm{THz}$, and the broad peak at $13.2 \mathrm{THz}$ is at the same level for both models. Above $14 \mathrm{THz}$, the narrow peak at $14.8 \mathrm{THz}$ dominates and the gain at higher frequency shifts is lower than for the analytical models. In conclusion, we would expect to have contribution to the noise from the peak at $14.8 \mathrm{THz}$ when the measured Raman gain is used in the simulations, but not when the analytical models are used, for which the main 13.2 THz peak dominates.

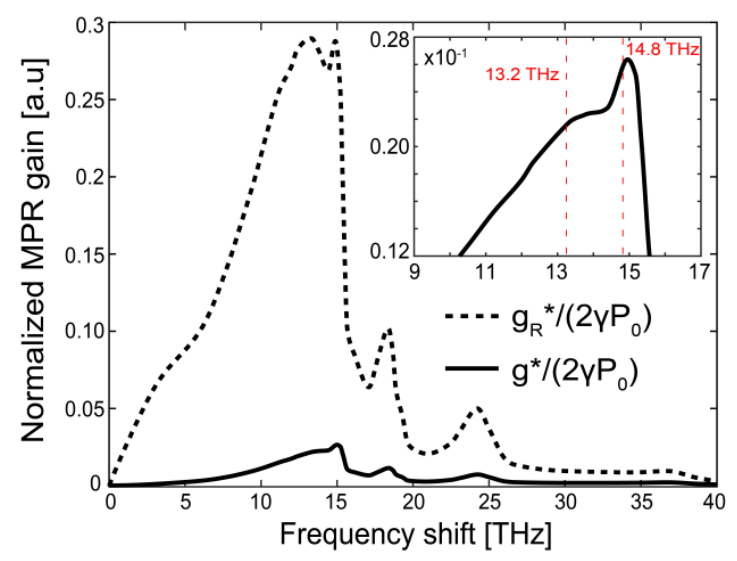

Fig. 2. Normalized MPR gain profile for the measured Raman gain and the parameters used in the simulation (solid), The corresponding pure Raman gain, obtained for $K \rightarrow-\infty$, is shown as a dashed line. The inset shows a maximum around $14.8 \mathrm{THz}$ for the MPR gain. 


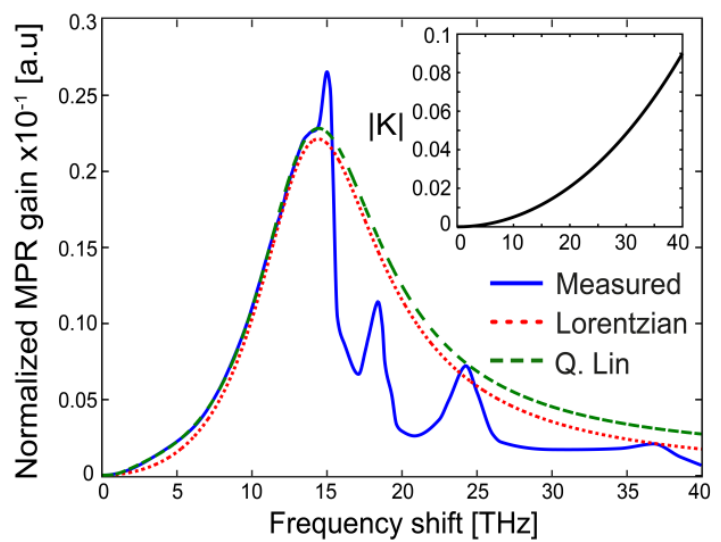

Fig. 3. Normalized MPR gain profile for the three Raman gain profiles in Fig 1(b): measured Raman gain (blue), Lorentzian (red) and Q. Lin (green) models. The inset shows the frequency dependence of $|K|$.

\section{B. Sub-picosecond pump pulses}

After the discussion of the influence of FWM on the effective Raman gain for the three Raman gain models, we perform simulations with the GNLSE for a sub-picosecond pulse length of $T_{F W H M}=600 \mathrm{fs}$ to investigate the effect of the Raman gain profile in the noise dynamics of the ANDi SC.

Figure 4 shows the evolution of the spectrum and the coherence obtained when using the Raman gains displayed in Fig. 1(b). For all cases, the SC evolves similarly initially and is fully generated already after $10 \mathrm{~cm}$ because the OWB distance [7] is short $L_{O W B}=7.03 \mathrm{~cm}$ for our conditions. After about $30 \mathrm{~cm}$, the long wavelength part of the SC spectrum develops spectral fluctuations for the measured Raman gain, whereas it continues to be smooth for the two analytical models. A spectral dip develops at around $850 \mathrm{~nm}$ after $50 \mathrm{~cm}$ for all models and another one develops at around $1150 \mathrm{~nm}$ after about $1 \mathrm{~m}$ for the measured Raman gain.

In relation to the noise, the MPR gain length is around $7 \mathrm{~mm}$ and thus we would expect to have the first signature of noise within a few $\mathrm{cm}$ of propagation. However, the noise starts much later in the fiber at around $0.5 \mathrm{~m}$ as a result of the fast SPM broadening. The coherence evolves similarly for the two analytical Raman models and as expected it develops slightly later for the Lorentzian model, which has a slightly lower MPR gain than the Q. Lin model for all wavelengths (see Fig. 3). The noise is seen to start on the short wavelength side close to the maximum blue SPM peak, as was also observed and discussed by Heidt et al. using the Q. Lin model [8].

However, when the measured Raman is used the evolution is different. At short distances around $0.5 \mathrm{~m}$, coherence is maintained at long wavelengths even though there are spectral fluctuations, as seen in Fig. 4(e-f). After more propagation, decoherence starts in the short wavelength side slightly later than with the analytical models. However, after around $80 \mathrm{~cm}$, decoherence develops in the long wavelength side of the spectrum, which is not observed with the analytical models. Therefore, the coherence degradation for wavelengths above the pump is faster when using the measured Raman gain in the simulations. From the analysis above we would anticipate this to be due to the strong peak in the MPR gain at $14.8 \mathrm{THz}$ (see Fig. 3), which is only present for the measured Raman gain profile and as we will show later this is also the case.

To look a bit deeper into the properties of the SC and coherence spectra we show in Fig. 5 single-shot spectrograms at $0.5 \mathrm{~m}, 1.0 \mathrm{~m}$, and $2.0 \mathrm{~m}$ propagation. The classical S-shape of the ANDi SC spectrum $[2,6]$ is seen in all spectrograms. We observe that at $0.5 \mathrm{~m}$, spectral fluctuations have appeared on the short wavelength side below the pump at around $800 \mathrm{~nm}$ for all the gain profiles Furthermore, the measured Raman gives also spectral fluctuations at longer wavelengths than the pump, not present with the analytical models. After $1 \mathrm{~m}$ of propagation, the fluctuation at short wavelengths spreads towards longer wavelengths and reached the pump wavelength for all the models. The long wavelength fluctuations have also spread for the measured Raman gain, but are still not present for analytical models. In the time domain, the interference is only located in the trailing part of the pulse for the analytical models. For the measured Raman, not only the trailing edge but also the leading edge of the pulse is corrupted.

At $2 \mathrm{~m}$ the SC spectrum has developed fluctuations across the whole bandwidth for the measured Raman gain, except below $700 \mathrm{~nm}$, which corresponds to the slowest trailing edge of the pulse in the time domain. In contrast, the fluctuations generated slowly from the $800 \mathrm{~nm}$ region with the analytical models have only spread to about $1300 \mathrm{~nm}$, so a smooth spectrum is still visible at longer wavelengths for these models.

The conclusion is that the $\mathrm{Q}$. Lin and Lorentzian models give similar behavior in the frequency and time domains (slightly noisier for the Q. Lin model), whereas using the measured Raman model the SC develops more fluctuations for wavelengths above the pump than the analytical models.

To investigate the effect of the Raman peak at $14.8 \mathrm{THz}$, we used the analytical multi-resonance model described in [25], which is a good approximation to the measured Raman gain profile across the whole spectrum and more importantly, for which the peaks can be easily individually removed. Due to its many resonances and consequently long expression, we refrain from giving it here, but refer the reader to [25]. In Fig. 6 we show the average spectrum (red), the spectral fluctuations (grey), and the coherence (blue) at $2 \mathrm{~m}$ obtained with the full model (a) and the full model without the $14.8 \mathrm{THz}$ peak (b). We also show the measured Raman gain model in Fig. 6(c). We see that when the peak at $14.8 \mathrm{THz}$ is removed (Fig. 6(b)), the noise and spectral fluctuations in the long wavelength side of the spectrum vanish, which confirms that they are indeed caused by the $14.8 \mathrm{THz}$ peak. The other secondary peaks above $14.8 \mathrm{THz}$ also contribute to the noise and spectral fluctuations, but with much less efficiency, as their gain is low.

After identifying the $14.8 \mathrm{THz}$ peak as the main responsible cause for coherence degradation in the long wavelength side of the SC, we now investigate the mechanism that leads to this additional noise dynamics. For this, the main features of the $14.8 \mathrm{THz}$ peak are examined: (a) the high MPR gain and (b) the spectral width of the peak. First of all, it is straightforward to understand that a higher gain results in faster decoherence, and therefore decreasing the gain of the 14.8 $\mathrm{THz}$ will reduce the coherence degradation at long wavelengths. For instance, when the gain of the $14.8 \mathrm{THz}$ peak is zero - corresponding to the case where the peak is removed (Fig. 6(b)) - the decoherence at long wavelength ceases.

The second important feature is the spectral width of the resonance peak. Narrow spectral resonances with sufficiently high gain result in longer temporal decay of the Raman response. Figure 7 shows the Raman response in the time domain when the $14.8 \mathrm{THz}$ peak is included (blue) or removed (red), which corresponds to the Raman gain used in the simulations in Fig. 6(a) and (b), respectively. We can see that the inclusion of the spectrally narrow $14.8 \mathrm{THz}$ peak results in a longer temporal decay of the Raman response as expected. The effect of the longer temporal decay on the noise generated at long wavelengths can be explained as follows: 

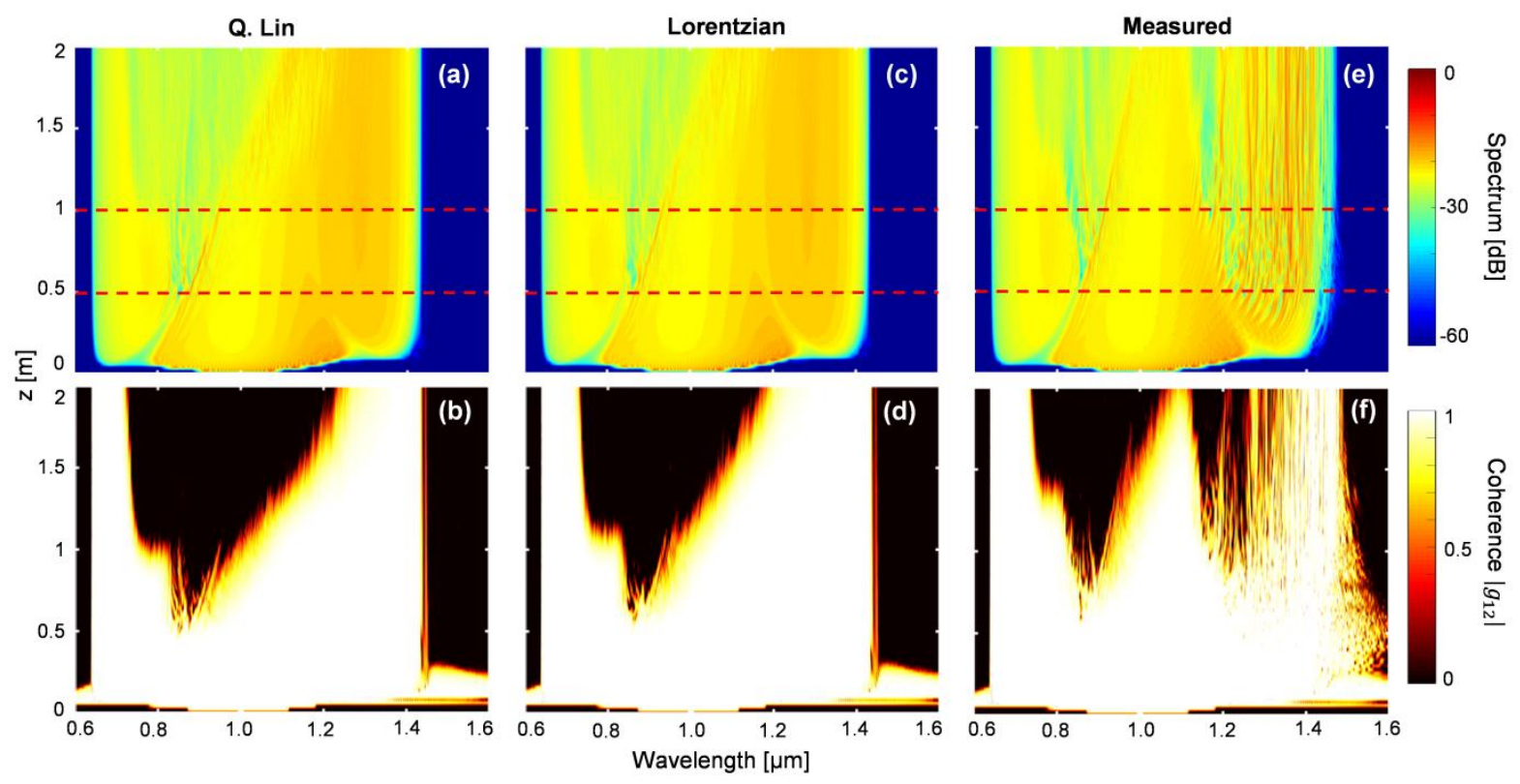

Fig. 4. Mean SC evolution and spectral coherence (for an ensemble of 20 simulations) over $2 \mathrm{~m}$ for $600 \mathrm{fs}$ pump at $1 \mu \mathrm{m}$ and $100 \mathrm{~kW}$ input peak power using the three different Raman gain profiles in Fig. 1(b). (a, b) Q. Lin model, (c, d) Lorentzian model and (e, f) measured Raman gain.
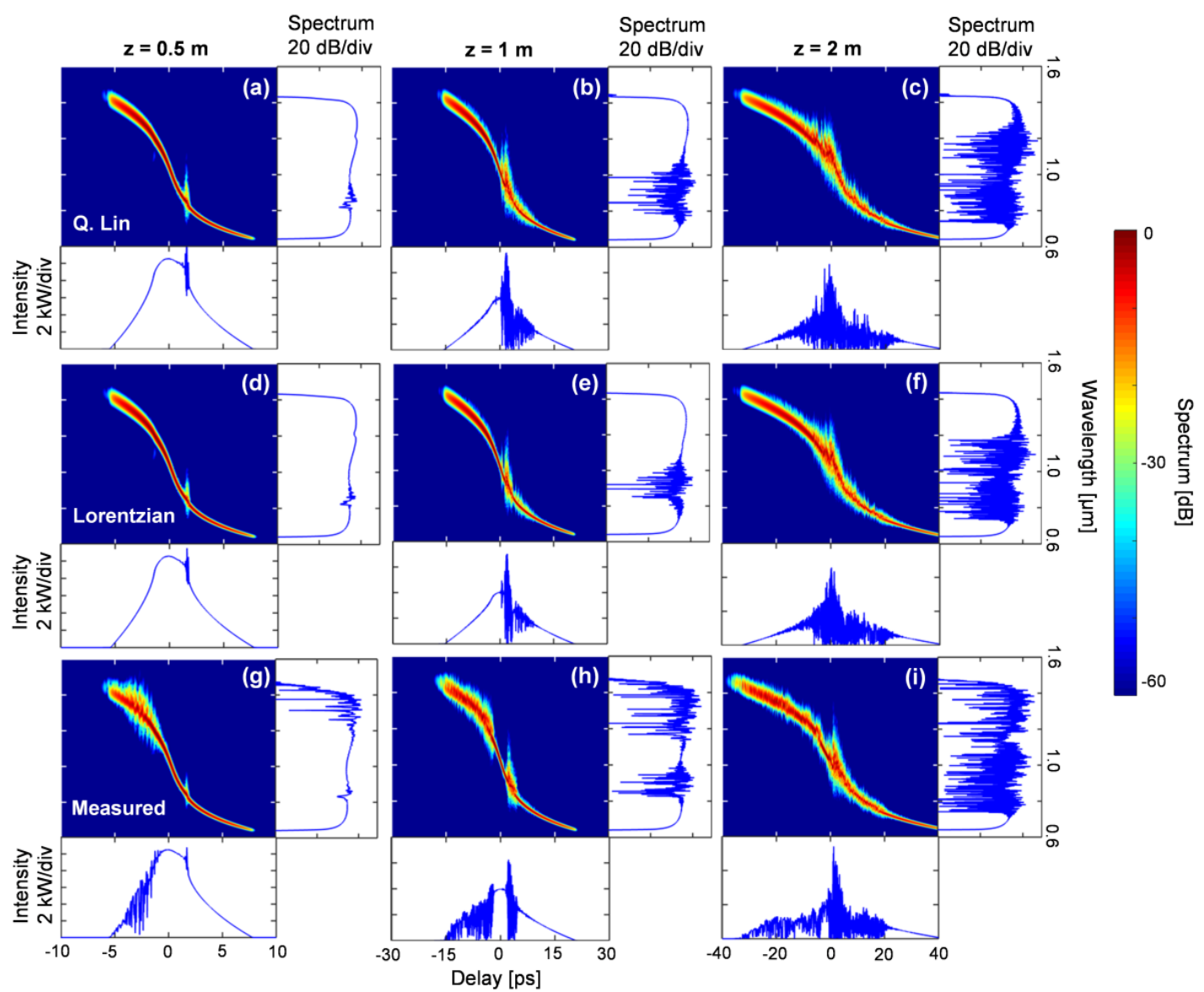

Fig. 5. Single-shot SC spectrograms for 600 fs pump at $1 \mu \mathrm{m}$ and $100 \mathrm{~kW}$ input peak power using the three different Raman gain profiles in Fig. 1(b). (a-c) Q. Lin model, (d-f) Lorentzian model and (g-i) measured Raman gain. Each column corresponds to a fixed propagation distance $(0.5 \mathrm{~m}, 1 \mathrm{~m}$ and $2 \mathrm{~m})$. 

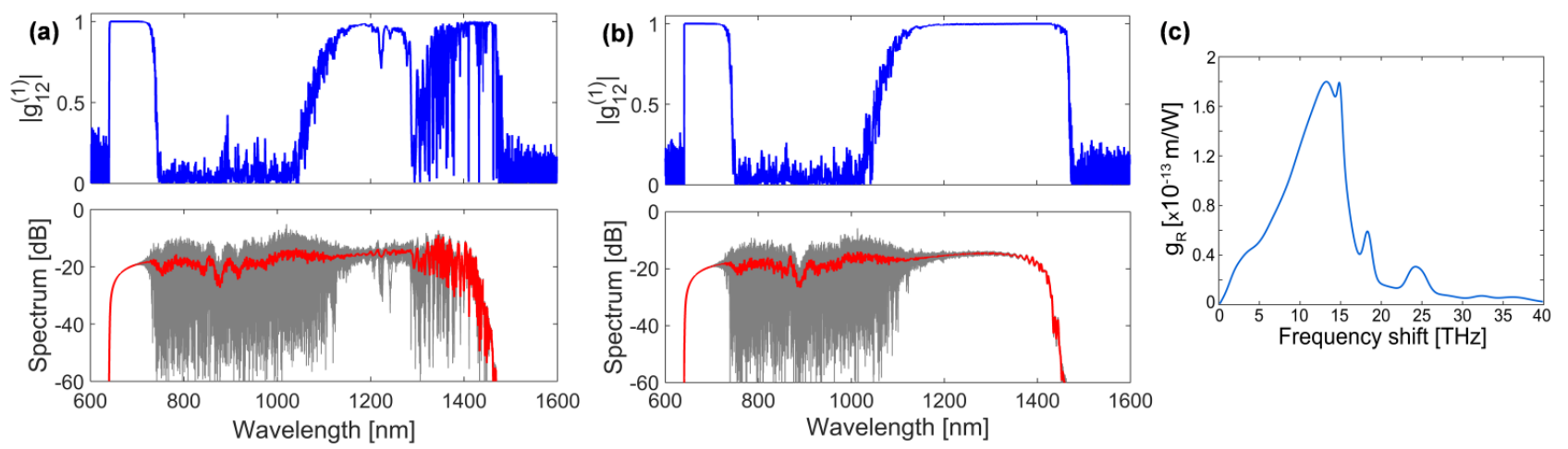

Fig. 6. Mean SC spectrum (red), spectral fluctuations (grey) and spectral coherence (blue) at $2 \mathrm{~m}$ for $600 \mathrm{fs}$ pump at $1 \mu \mathrm{m}$ and $100 \mathrm{~kW}$ input peak power using (a) the full model for the measured Raman and (b) the model without the peak at $14.8 \mathrm{THz}$. (c) full model for the measured Raman given in [25].

As Raman components are generated from the outermost SPM peaks on both sides of the spectrum, we would expect to have noise in both sides of the spectrum, however, this is not the case when the analytical Raman models are used. This can be explained by the fact that the generated Raman components are located in two completely different regions. In the short wavelength side, the Raman Stokes component, which is the strongest, lies in the SPM region close to the outermost SPM peak, whereas in the long wavelength side, the Raman Stokes line lies in a region with lower power than the SPM region. Therefore, the generation of a Stokes line in the SPM region, which has more power, together with the faster dynamics in the short wavelength side allows for more noise to be accumulated in this region than at the long wavelength side of the SC. However, the noise dynamics can change when a spectrally narrow peak with high MPR gain, such as the $14.8 \mathrm{THz}$ peak, is included in the Raman gain. The high MPR gain (18\% higher than the broad $13.2 \mathrm{THz}$ peak) results in a faster growth of the Raman noise, while the longer temporal decay of the Raman response can lead to further accumulation of noise, which together cause the decoherence of the long wavelength side of the SC.

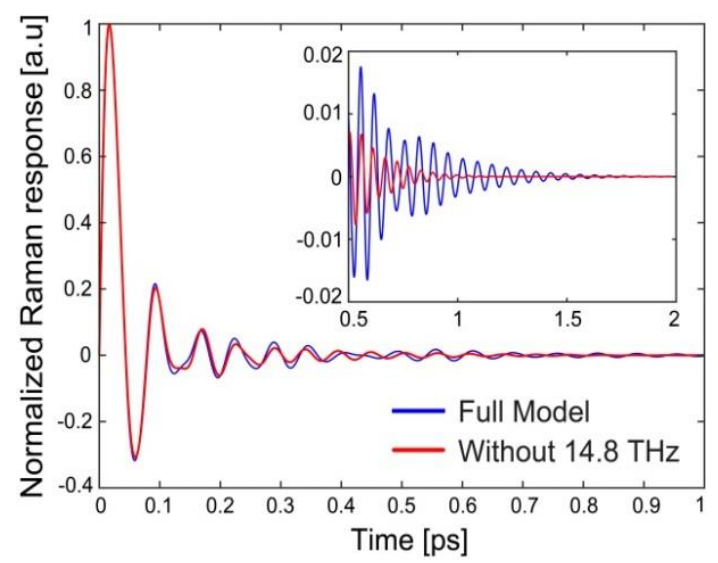

Fig. 7. Normalized Raman response in the time domain, corresponding to the full (including the $14.8 \mathrm{THz}$ peak) multi-resonance Raman model (blue) and when the $14.8 \mathrm{THz}$ peak is removed (red). The inset shows the longer decay time of the Raman response when the narrow resonance peak at $14.8 \mathrm{THz}$ is included.

To investigate the effect of the spectral width of the resonance peaks, the multi-resonance model [25] is used again, but in this case the $24 \mathrm{THz}$ peak is numerically enhanced to have the same MPR gain as the $14.8 \mathrm{THz}$ peak in Fig. 3. Afterwards, the $14.8 \mathrm{THz}$ peak is removed in order to study the effect of the $24 \mathrm{THz}$ peak alone. The choice to investigate the $24 \mathrm{THz}$ peak is because it is easier to change its width without altering the $13.2 \mathrm{THz}$ peak. Figure 8(a) shows the two Raman gains used in the simulations to investigate the $24 \mathrm{THz}$ peak. The corresponding Raman response in the time domain (Fig. 8(b)) shows a longer decay time for the narrow (dark blue curves) than for the wide (light blue curves) $24 \mathrm{THz}$ peak. Simulations were then performed similar to Fig. 6 but with the Raman gain in Fig. 8(a). We observe that the decoherence at long wavelengths only surfaces for a sufficiently narrow $24 \mathrm{THz}$ peak (Fig. 8 (c)) and vanishes when the peak is wide (Fig. 8 (d)). This result suggests that there is a correspondence between narrow resonance peaks and the additional noise at long wavelengths. Moreover, the position of the narrow resonance peak seems to play a qualitatively minor role in the decoherence at long wavelengths, because this additional noise is present regardless of where the peak is located.

\section{Picosecond pump pulses}

In this subsection, we investigate the effect of the Raman gain on the noise dynamics for a longer $1.6 \mathrm{ps}$ pump pulse, but otherwise unchanged parameters, in order to examine the influence of the pump pulse length. We show in Fig. 9 the evolution of the spectrum and coherence as in Fig. 4 for 600 fs. In this case, the broadest SC is generated later in the fiber due to the longer OWB distance, $L_{O W B}=18.7 \mathrm{~cm}$, compared to the $600 \mathrm{fs}$ case. The MPR gain length is the same as in the $600 \mathrm{fs}$ case as it does not depend on the pulse length. However, the SPM and OWB dynamics is slower than in the subpicosecond case and thus the decoherence starts earlier. For all the Raman gain profiles the SC evolves now similarly along the fiber.

At around $17 \mathrm{~cm}$ the OWB has developed in the short wavelength side. In the long wavelength side, OWB happens again later due to the smaller slope of the dispersion, which makes the group velocity difference smaller between the pump and the outermost SPM peak than at the shorter wavelength side [8]. Consequently, we see that a Raman peak is being generated before OWB and this long wavelength Raman peak is introducing noise quickly covering the whole long wavelength regime.

The short wavelength noise around $800 \mathrm{~nm}$ is still observed, but now it appears after the long wavelength Raman peak being generated, which means that the noise dynamics is very similar for the three Raman gain profiles under study. We can then conclude that the specific contribution of the peak at $14.8 \mathrm{THz}$ is less important for the qualitative noise development than in the sub-picosecond pump case. 
(a)
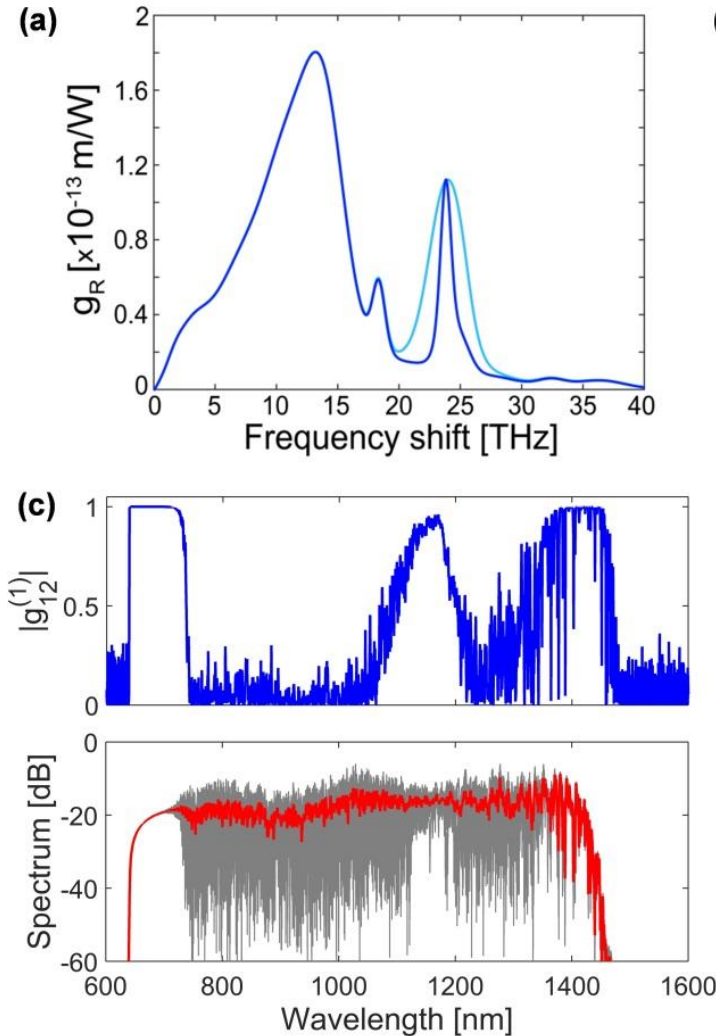

(b)
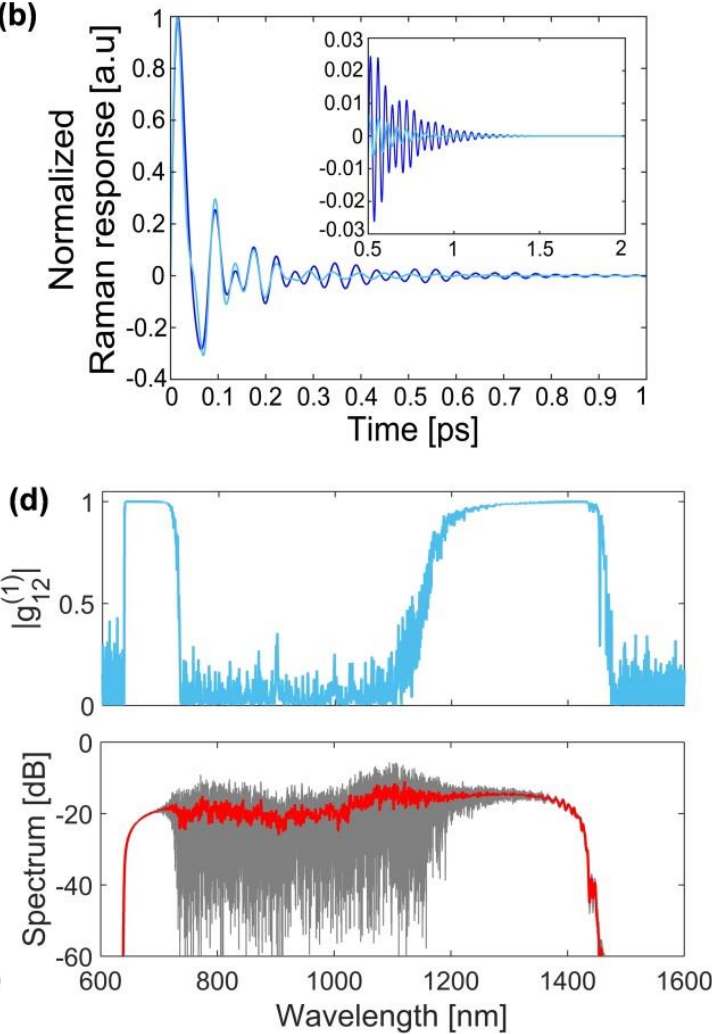

Fig. 8. (a) Pure Raman gain and (b) corresponding normalized Raman response time with numerically enhanced and wide (light blue) and narrow (dark blue) $24 \mathrm{THz}$ peak. The inset in (b) shows longer decay time for the narrower $24 \mathrm{THz}$ peak (dark blue). Mean SC spectrum (red), spectral fluctuations (grey) and spectral coherence (blue) at $2 \mathrm{~m}$ using the Raman gain with (c) the narrow and (d) the wide $24 \mathrm{THz}$ peak.
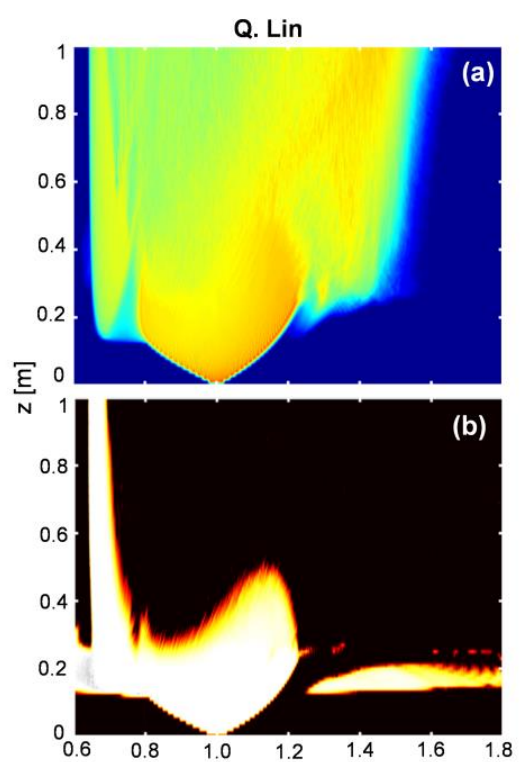
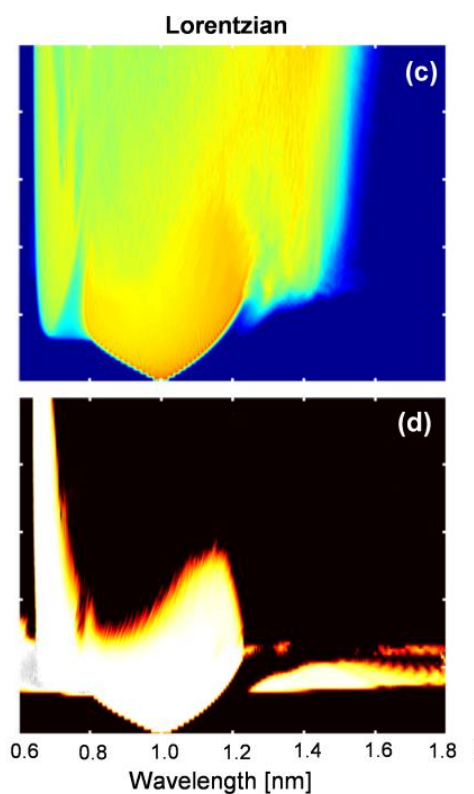
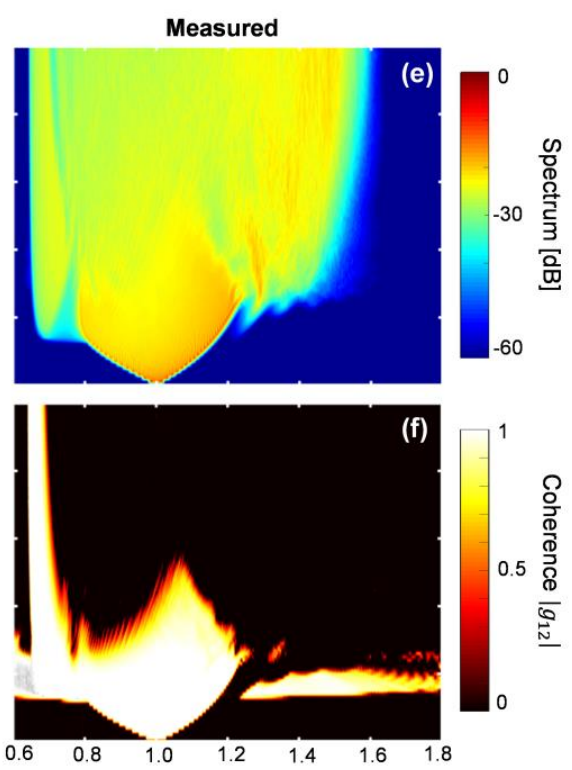

Fig. 9. Mean SC evolution and spectral coherence (for an ensemble of 20 simulations) over $1 \mathrm{~m}$ for $1.6 \mathrm{ps}$ pump at $1 \mu \mathrm{m}$ and $100 \mathrm{~kW}$ input peak power using the three different Raman gain profiles in Fig. 1(b). (a, b) Q. Lin model, (c, d) Lorentzian model and (e, f) measured Raman gain. 


\section{CONCLUSIONS}

We theoretically and numerically investigated the effect of the Raman gain profile on the noise performance of an SC generated in a standard ANDi silica fiber pumped close to the wavelength of maximum dispersion. We theoretically showed that when the conditions of Raman gain suppression by FWM are satisfied, the frequency dependence of the effective MPR gain can differ significantly from that of the pure Raman gain. The reduction of the MPR gain is more pronounced at frequency shifts below $13 \mathrm{THz}$, which means that the Boson peak at $3 \mathrm{THz}$ is completely suppressed. Most importantly, the frequency dependence of the FWM induced Raman gain suppression means that the measured MPR gain has its strongest localized peak at $14.8 \mathrm{THz}$ and not at $13.2 \mathrm{THz}$ as in the pure Raman gain and the analytical Lorentzian and Q. Lin models of the Raman gain. The $14.8 \mathrm{THz}$ peak is $18 \%$ stronger than the $13.2 \mathrm{THz}$ gain value, which can significantly change the noise performance of ANDi SC generation.

For sub-picosecond pump pulses (600 fs in our investigations), our numerical simulations confirmed that a significantly different noise dynamics is found using the measured Raman gain in the simulations, compared to the commonly used analytical Raman models. In particular, the $14.8 \mathrm{THz}$ peak introduces noise in the long wavelength regime above the pump not present in the analytical models.

We also showed that the additional noise at long wavelengths due to the $14.8 \mathrm{THz}$ peak is related not only to its high gain, but also to its spectrally narrow width. As a result of the longer decay time of the Raman response in the time domain and the consequent accumulation of noise, decoherence in the long wavelength side of the SC is observed for sub-picosecond pump pulses.

For picosecond pulses (1.6 ps in our investigations), due to the slower SC evolution compared to the sub-picosecond pulses, the Raman lines develop faster and have a stronger contribution to the noise at long wavelengths. In this case, we found that similar noise performance was obtained using the measured Raman gain and the Lorentzian and Q. Lin analytical models.

To conclude, our results indicate that the use of the measured Raman gain of silica or a multi-resonance model that includes the 14.8 $\mathrm{THz}$ peak [25] should preferably be used in numerical modeling to calculate the noise performance of ANDi SC generation in silica fibers.

Funding. IBG and $\mathrm{OB}$ acknowledge financial support from Innovationsfonden (SHAPEOCT-4107-00011B); Det Frie Forskningsråd (DFF) (LOISE-4184-00532B), and Horizon 2020 Framework Programme (H2020) (GALAHAD-732613).

\section{REFERENCES}

1. N. Nishizawa and J. Takayanagi, "Octave spanning high-quality supercontinuum generation in all-fiber system," J. Opt. Soc. Am. B 24, 1786-1792 (2007).

2. A. M. Heidt, A. Hartung, G. W. Bosman, P. Krok, E. G. Rohwer, H. Schwoerer, and $\mathrm{H}$. Bartelt, "Coherent octave spanning near-infrared and visible supercontinuum generation in all-normal dispersion photonic crystal fibers," Opt. Express 19, 3775-3787 (2011).

3. M. Klimczak, G. Soboń, R. Kasztelanic, K. M. Abramski, and R. Buczyński, "Direct comparison of shot-to-shot noise performance of all normal dispersion and anomalous dispersion supercontinuum pumped with sub-picosecond pulse fiber-based laser," "Sci. Rep. 6, 19284 (2016).

4. J. M. Dudley, G. Genty, and S. Coen, "Supercontinuum generation in photonic crystal fiber," Rev. Mod. Phys. 78, 1135-1184 (2006).

5. G. Agrawal, Nonlinear Fiber Optics (Academic, 2013).
6. A. M. Heidt, "Pulse preserving flat-top supercontinuum generation in allnormal dispersion photonic crystal fibers," J. Opt. Soc. Am. B 27, 550559 (2010).

7. C. Finot, B. Kibler, L. Provost, S. J. Wabnitz, "Beneficial impact of wavebreaking for coherent continuum formation in normally dispersive nonlinear fibers," J. Opt. Soc. Am. B 25, 1938-1948 (2008).

8. A. M. Heidt, J. S. Feehan, J. H. V. Price, and T. Feurer, "Limits of coherent supercontinuum generation in normal dispersion fibers," J. Opt. Soc. Am. B 34, 764-775 (2017).

9. I. B. Gonzalo, R. D. Engelsholm, M. P. Sørensen, and O. Bang, "Polarization noise places severe constraints on coherence of all-normal dispersion femtosecond supercontinuum generation," Sci. Rep. 8, 6579 (2018).

10. J. Hult, "A Fourth-Order Runge-Kutta in the Interaction Picture Method for Simulating Supercontinuum Generation in Optical Fibers," J. Lightwave Technol. 25, 3770-3775 (2007).

11. J. Zhou, K. Tajima, K. Nakajima, K. Kurokawa, C. Fukai, T. Matsui, and I. Sankawa, "Progress on low loss photonic crystal fibers," Optical Fiber Technology 11, 101-110 (2005).

12. Q. Lin and G. P. Agrawal, "Raman response function for silica fibers," Opt. Lett. 31, 3086-3088 (2006).

13. R. H. Stolen and E. P. Ippen, "Raman gain in glass optical waveguides," Appl. Phys. Lett., 22, 276-281 (1973).

14. M. H. Frosz, "Validation of input-noise model for simulations of supercontinuum generation and rogue waves," Opt. Express 18, 1477814787 (2010).

15. J. M. Dudley and S. Coen, "Coherence properties of supercontinuum spectra generated in photonic crystal and tapered optical fibers," Opt. Lett. 27, 1180-1182 (2002).

16. S. T. Sørensen, O. Bang, B. Wetzel, and J. M. Dudley, "Describing supercontinuum noise and rogue wave statistics using higher-order moments," Opt. Comm. 285, 2451-2455 (2012).

17. R. H. Stolen, J. P. Gordon, W. J. Tomlinson, and H. A. Haus, "Raman Response Function of Silica-core Fibers," J. Opt. Soc. Am. B 6, 11591166 (1989).

18. R. W. Hellwarth, "Third-order optical susceptibilities of liquids and solids," Prog. Quantum Electron. 5, 1-68 (1977).

19. K. J. Blow and D. Wood, "Theoretical description of transient stimulated Raman scattering in optical fibers," IEEE J. Quantum Electron. 25, 26652673 (1989).

20. D. J. Dougherty, F. X. Kärtner, H. A. Haus, and E. P. Ippen, "Measurement of the Raman gain spectrum of optical fibers," Opt. Lett. 20, 31-33 (1995).

21. K. Rottwitt and J. H. Povlsen, "Analyzing the Fundamental Properties of Raman Amplification in Optical Fibers," J. Lightwave Technol. 23, 3597 3605 (2005).

22. N. Bloembergen and Y. R. Shen, "Coupling Between Vibrations and Light Waves in Raman Laser Media," Phys. Rev. Lett. 12, 504-507 (1964).

23. E. A. Golovchenko and A. N. Pilipetskii, "Unified analysis of four-photon mixing, modulational instability, and stimulated Raman scattering under various polarization conditions in fibers," J. Opt. Soc. Am. B 11, 92-101 (1994).

24. F. Vanholsbeeck, P. Emplit and S. Coen, "Complete experimental characterization of the influence of parametric four-wave mixing on stimulated Raman gain," Opt. Lett. 28, 1960-1962 (2003).

25. D. Hollenbeck and C. D. Cantrell, "Multiple-vibrational-mode model for fiber-optic Raman gain spectrum and response function," J. Opt. Soc. Amer. B, 19, 2886-2892 (2002). 